\title{
Correction to: A study of thermal controls in rapidly developing city using remotely sensed satellite data: spatiotemporal perspective
}

\author{
Khalid Mahmood $^{1}$ D . Syed Ahmed Raza ${ }^{2} \cdot$ Syeda Yuman Fatima ${ }^{1}$
}

Published online: 8 February 2021

(c) Institute of Geophysics, Polish Academy of Sciences \& Polish Academy of Sciences 2021

\section{Correction to: Acta Geophysica}

https://doi.org/10.1007/s11600-020-00535-9

In the article "A Study of Thermal Controls in Rapidly Developing City Using Remotely Sensed Satellite Data: Spatio-Temporal Perspective" by Mahmood et al. (Journal of Acta Geophysica, advance online publication, January, 2021, http://link.springer.com/article/10.1007/s11600-020-00535 -9) the affiliation of first (Khalid Mahmood) and third (Syeda Yuman Fatima) authors has been wrongly stated, the correct affiliation of both the authors is "Remote Sensing, GIS and Climatic Research Lab (National center for GIS and Space Applications), Department of Space Science, University of the Punjab, Lahore, Pakistan".

The original article has been corrected.

The original article can be found online at https://doi.org/10.1007/ s11600-020-00535-9.

Khalid Mahmood

khalid.spsc@pu.edu.pk

1 Remote Sensing, GIS and Climatic Research Lab (National Center for GIS and Space Applications), Department of Space Science, University of the Punjab, Lahore, Pakistan

2 Department of Space Science, University of the Punjab, Lahore, Pakistan 